Some Reflections on Combining Meta-Analysis and Structural Equation Modeling

Mike W.-L. Cheung

National University of Singapore

Please cite this paper as:

Cheung, M.W.-L. (in press) Some reflections on combining meta-analysis and structural equation modeling. Research Synthesis Methods.

Author Note

Mike W.-L. Cheung, Department of Psychology, National University of Singapore. This research was supported by the Academic Research Fund Tier 1 (FY2017-FRC1-008) grant from the Ministry of Education, Singapore.

Correspondence concerning this article should be addressed to Mike W.-L. Cheung, Department of Psychology, Faculty of Arts and Social Sciences, National University of Singapore, Block AS4, Level 2, 9 Arts Link, Singapore 117570. Tel: (65) 6516-3702; Fax: (65) 6773-1843; E-mail: mikewlcheung@nus.edu.sg. 


\begin{abstract}
Meta-analysis and structural equation modeling (SEM) are two of the most prominent statistical techniques employed in the behavioral, medical, and social sciences. They each have their own well-established research communities, terminologies, statistical models, software packages, and journals (Research Synthesis Methods and Structural Equation Modeling: A Multidisciplinary Journal). In this paper, I will provide some personal reflections on combining meta-analysis and SEM in the forms of meta-analytic SEM (MASEM) and SEM-based meta-analysis. The critical contributions of Becker (1992), Shadish (1992), and Viswesvaran and Ones (1995) in the early development of MASEM are highlighted. Another goal of the paper is to illustrate how meta-analysis can be extended and integrated with other techniques to address new research questions such as the analysis of Big Data. I hope that this paper may stimulate more research development in the area of combining meta-analysis and SEM.
\end{abstract}

Keywords: meta-analysis, structural equation modeling, meta-analytic structural equation modeling, SEM-based meta-analysis 


\section{Some Reflections on Combining Meta-Analysis and Structural Equation Modeling}

Meta-analysis, a term coined by Gene Glass, represents "the statistical analysis of a large collection of analysis results” (p. 3). ${ }^{1}$ After Glass's introduction of it to the scientific communities, meta-analysis has become the standard methodology in synthesizing research findings in the social, behavioral, educational, and medical sciences. The special issue on the origins of modern meta-analysis edited by Shadish ${ }^{2}$ gives several personal perspectives from some of the main contributors to the development of meta-analysis. ${ }^{3},{ }^{4}, 5$ (See also. ${ }^{6}$ ) Shadish and Lecy ${ }^{7}$ provide a history and a bibliometric analysis of the impact of meta-analysis in various fields.

Structural equation modeling (SEM) ${ }^{8},{ }^{9}$ which integrates various multivariate techniques in psychology, sociology, and economics, is a statistical technique to test complex models with latent and observed variables. Theoretical constructs or latent variables are linked to the observed variables through a measurement model. Hypothesized relationships among the observed and latent variables are specified via structural models. Researchers may, for example, hypothesize how the observed variables are related by specifying a path model. The estimated path coefficients inform the researchers how the variables affect other variables. Another popular model is confirmatory factor analysis, which links the observed variables to the latent variables. Researchers may study the relationships among the latent variables after controlling for measurement errors. Exact and approximate test statistics can be used to evaluate the consistency of the proposed model against the data. ${ }^{10}$ SEM provides a powerful tool to test "causal” models with observational data. Cudeck, Du Toit, and Sörbom ${ }^{11}$ and Matsueda ${ }^{12}$ offer several historical accounts of the origins and development of SEM.

Both Peter Bentler and Karl Jöreskog, key contributors to SEM in the early decades, received the Award for Distinguished Scientific Applications of Psychology "for [their] development of models, statistical procedures, and [computer algorithms] for structural equation modeling (SEM) that changed the way in which inferences are made from observational data” (p. 769). ${ }^{13},{ }^{14}$ As an important aside, Robert Rosenthal, John Hunter, and Frank Schmidt, all are well-known in the meta-analysis community, had also previously received the same award.

Researchers have used several slightly different terms in the literature for the class of techniques used to combine meta-analysis and SEM for testing theoretical models, e.g., model-driven meta-analysis, ${ }^{15}$ model-based meta-analysis, ${ }^{16}$ meta-analytic path analysis, ${ }^{17}$ meta-analysis of factor analysis, ${ }^{18},{ }^{19}$ meta-analytical structural equations analysis, ${ }^{20}$ path analysis of meta-analytically derived correlation matrices, ${ }^{21}$ structural equation modeling of a 
meta-analytic correlation matrix, ${ }^{22}$ path analysis based on meta-analytic findings, ${ }^{23}$ and analyzing matrices of meta-analytic correlations. ${ }^{24}$ The generic term "meta-analytic SEM" or "MASEM" is used here to represent this class of techniques. Apart from MASEM, there is another class of methods known as SEM-based meta-analysis, which uses the SEM framework to conduct univariate, multivariate, and three-level meta-analyses.

In this paper, I will give my personal reflections on synthesizing meta-analysis and SEM. The critical contributions of Becker, ${ }^{25}$ Shadish, ${ }^{26}$ and Viswesvaran and Ones ${ }^{27}$ in the early methodological development of MASEM are highlighted. Another goal in writing this paper is to show how meta-analysis can be extended and integrated with other techniques to address new research questions.

\section{The Beginning of MASEM}

SEM is a favorite technique among researchers in the social and behavioral sciences, who use it to test hypothesized models with observational data. When a number of SEM studies have been produced on similar topics, researchers may want to combine these studies with a meta-analysis. Becker and Schram ${ }^{15}$ and Viswesvaran and Ones ${ }^{27}$ reviewed some early applications of MASEM in psychology. These include, for example, the work of Harris and Rosenthal, ${ }^{28}$ Schmidt, Hunter, and Outerbridge, ${ }^{29}$ and Premack and Hunter. ${ }^{30}$

There are two stages of conducting a MASEM. In the first stage, the correlation matrices are meta-analytically combined to form a pooled correlation matrix. In the second stage, the pooled correlation matrix is used to fit structural equation models. Likelihood ratio statistic and various goodness-of-fit indices are used to evaluate the appropriateness of the proposed models.

The first stage of the analysis is merely a multivariate meta-analysis on the correlation matrices, which is already well known in the meta-analytic literature. ${ }^{31}, 32$ If researchers are only interested in making inferences on the pooled correlation matrix, existing meta-analytic techniques are sufficient for use. However, almost all researchers who conduct MASEM are interested in fitting theoretical models in SEM. The literature on SEM shows that there are statistical issues when the pooled correlation matrix is treated as an observed covariance matrix in fitting structural equation models. ${ }^{33}, 34,35,{ }^{36}$ Simulation results also support the view that these concerns cannot be ignored. ${ }^{37},{ }^{38},{ }^{39}$ MASEM is complex in that its procedures involve both meta-analysis and SEM. Researchers conducting MASEM should, therefore, be aware of issues involving both meta-analysis and SEM.

The papers by Becker, ${ }^{25},{ }^{40}$ Shadish, ${ }^{26}$ and Viswesvaran and Ones ${ }^{27}$ were probably the first few methodological papers on MASEM. They played a significant role in my learning of MASEM. Shadish ${ }^{26}$ gave an introduction to integrating meta-analysis with SEM. Although he 
did not give the statistical details of the process, he discussed many vital issues in combining meta-analysis and SEM to test causal mediating processes, using published examples. Most importantly, he examined the roles of the within-study variation (average correlation matrix) and the between-study variation (heterogeneity of the correlation coefficients) in combining meta-analysis and SEM.

Becker $^{25}$ proposed a fixed- and a random-effects model for MASEM. She used the generalized least squares (GLS) and methods of moment estimators to combine correlation matrices in the first stage of the analysis. Becker and Schram ${ }^{15}$ later extended it to a randomeffects model using the EM algorithm to estimate the fixed- and random effects. In the second stage of the analysis, Becker proposed fitting regression or path models with the pooled correlation matrix. Becker used matrix partitioning to calculate the parameter estimates of the regression or path models. Standard errors in the parameter estimates of the structural equation models are calculated using the multivariate delta method.

Becker's model provides the foundation for my work. However, there are two practical limitations to her approach that present challenges to applied researchers. First, the models that can be fitted are limited to regression and path models. Applied researchers are usually more interested in testing complex path or full structural equation models; those model fit can be accessed by various goodness-of-fit indices. It is not straightforward to calculate the goodness-of-fit indices in Becker's approach. Second, the implementations of the analyses are not trivial for many applied researchers, especially when researchers want to test the equality of some path coefficients. Researchers may not have the necessary skills to write their programs to run the analyses. Since SEM is ideal for fitting linear models, I extended and implemented Becker's approach into the SEM framework (see the discussion on this later in this paper).

Viswesvaran and Ones ${ }^{27}$ suggested an alternative approach to conducting MASEM. They used Hunter and Schmidt's ${ }^{41}$ method to combine the correlation coefficients in the first stage of the analysis. In the second stage of the analysis, the pooled correlation matrix is directly used in fitting structural equation models. As the elements of the combined correlation matrices are usually based on different sample sizes, Viswesvaran and Ones proposed using the harmonic mean of the sample sizes in SEM.

Viswesvaran and Ones's approach is fundamentally equivalent to running two separate analyses - one meta-analysis and one SEM. No new statistical theories or software are involved. Because of its ease of use, the approach of Viswesvaran and Ones appeals to applied researchers. Sheng, Kong, Cortina, and $\mathrm{Hou}^{24}$ reported that more than $90 \%$ of MASEM applications used Viswesvaran and Ones's approach. Rosopa and Kim ${ }^{42}$ also made similar observations in their review of MASEM applications used in human resource 
management, industrial-organizational psychology, and allied fields. It is fair to say that Viswesvaran and Ones’s approach has popularized MASEM in substantive areas.

\section{Further Development of MASEM}

I was a graduate student under Wai Chan, who was a student of Peter Bentler, when I first learned MASEM from Becker, ${ }^{25}$ Shadish, ${ }^{26}$ and Viswesvaran and Ones. ${ }^{27}$ My training in statistics was primarily in the field of SEM. I acquired my knowledge of meta-analysis mainly by reading Hedges and Olkin. ${ }^{43}$ Because of my background in SEM, I had some issues with how structural equation models were fitted in Viswesvaran and Ones’s approach.

There are at least two problems with Viswesvaran and Ones's approach (see Section 7.2.1 of Cheung ${ }^{44}$ for more details). First, the elements of the pooled correlation matrix are obtained by aggregating correlation coefficients from different studies. This process is similar to pairwise deletion in SEM. Simulation studies ${ }^{33}$ have shown that the test statistics and standard errors of the parameters in SEM are likely biased. The use of one sample size, the harmonic mean of the sample sizes, does not provide correct statistical inferences for all of the elements in the pooled correlation matrix. The precision of some estimated correlation elements is over-estimated, whereas that of others is under-estimated. Second, the pooled correlation matrix is treated as if it were a covariance matrix in fitting structural equation models. Cudeck ${ }^{35}$ warned researchers against using correlation matrices as covariance matrices in SEM because the test statistics and standard errors could be incorrect.

Becker's approach has essentially resolved these issues. However, her approach is only limited to fitting path models without latent variables. In my $\mathrm{PhD}$ dissertation, I extended her approach to a "pure" SEM framework; 45 that is, to use SEM in both stages of MASEM. I proposed using a multiple-group SEM to combine the correlation matrices under the fixed-effects model in the first stage of the analysis. This is similar to Becker's fixedeffects approach except that in my approach there is no need to calculate the sampling variance-covariance matrix of the correlation matrices, which are treated as known in Becker's approach. The central idea is to consider the fixed-effects meta-analysis as a multiple-group SEM. The correlation matrices and standard deviations are treated as sample statistics with their own sampling uncertainty. ${ }^{38}$ Linear and non-linear constraints can be imposed in SEM. The common correlation matrix is estimated by imposing appropriate equality constraints on the correlation coefficients. The sampling variance-covariance matrices on the correlation matrices of the studies are not even estimated in the analysis.

In the second stage of the analysis, I fitted the structural equation models with the inverse of the sampling variance-covariance matrix of the correlation coefficients as the weight matrix. I did this using the asymptotically distribution-free (ADF) estimation 
method, ${ }^{46}$ which is also known as the weighted least squares (WLS) estimation method in SEM. The ADF estimation method is a generalization of Becker's method to fit structural equation models with both observed and latent variables. ${ }^{47}$ Since my approach uses SEM to conduct the analyses, such models as regression, path, or full structural equation models can be tested. Statistical inferences with the likelihood ratio statistics, goodness-of-fit indices, and standard errors of the parameter estimates in SEM are available. Linear and non-linear constraints can also be imposed on any parameters in SEM. Researchers may test whether some of the path coefficients are the same. Computer simulation studies have shown that this approach has good performance under the fixed-effects model. I called this approach "TwoStage Structural Equation Modeling” (TSSEM). ${ }^{38}{ }^{48}$

The TSSEM approach addresses several issues in Viswesvaran and Ones. First, a correlation structure model is used to analyze the pooled correlation matrix; there is no need to treat the correlation matrix as if it were a covariance matrix. Second, the precision of the combined correlation matrix is incorporated into the second stage of the analysis; with the use of the ADF estimation method, the ad-hoc use of sample size does not affect the statistical inferences and standard errors. The main limitation at the time that I conceived this approach, however, was that it was based on the fixed-effects model.

During this period, several researchers were also independently working on the MASEM methodology. Hafdahl ${ }^{49}$ studied how to meta-analyze findings for exploratory factor analysis, which is useful when there were no precise theoretical structures on the measurement models. S. F. Cheung ${ }^{50}$ and Furlow and Beretvas ${ }^{37}$ investigated how missing data affect the performance of MASEM. This topic is important because complete data are rare in MASEM. It was found that the parameter estimates are unbiased when the correlation coefficients are either missing completely at random or missing at random. When the missing occurs not at random, the parameter estimates may be biased.

\section{Recent Developments in MASEM}

To address the limitations of using the fixed-effects model in the TSSEM approach, I extended it to the random-effects model using a multivariate random-effects meta-analysis employing the SEM approach in the first stage of the analysis. ${ }^{51}$ Similar to other randomeffects meta-analyses, the sampling covariance matrices of the correlation matrices have to be estimated and assumed known. Olkin and Siotani ${ }^{52}$ provided the formula to compute these estimates (see Becker ${ }^{31}$ for the historical contributions of Olkin to multivariate metaanalysis). The assumption of known sampling covariance matrices is questionable when the sample sizes are small. Ad-hoc suggestions for using the average sample size to calculate the sampling covariance matrix seem to work well in simulation studies. ${ }^{53},{ }^{37},{ }^{54}$ 
The second stage of the analysis is still based on the ADF estimation method in SEM. These procedures are implemented in the metaSEM package ${ }^{55}$ in the R statistical platform, ${ }^{56}$ which is freely available to users. Later, I summarized these works on the subject of combining meta-analysis and SEM in my book. ${ }^{44}$ Suzanne Jak received a grant to work with me as a post-doctoral fellow for one year and wrote a brief introduction on how to conduct MASEM. ${ }^{57}$

One recent debate in MASEM is how to conceptualize heterogeneity in MASEM. ${ }^{58}$ Cheung and Cheung ${ }^{59}$ outlined two possible conceptualizations of random effects in MASEM - correlation-based versus parameter-based MASEM. In correlation-based MASEM, the correlation matrices are the effect sizes for the meta-analysis. The majority of the published works on MASEM are based on this approach. On the other hand, parameter-based MASEM treats the estimated parameters in SEM as the effect sizes for the meta-analysis. Only a few studies use this approach. ${ }^{60}, 61,62,63,{ }^{64},{ }^{65},{ }^{66}$ Correlation-based MASEM conceptualizes the heterogeneity at the correlation level, because there is only one structural equation model. Parameter-based MASEM considers the parameters in the structural equation model to be random. In other words, the parameters in the structural equation models have a multivariate normal distribution similar to that of the random effects of the correlation matrix in correlation-based MASEM.

Yu, Downes, Carter, and O’Boyle ${ }^{67}$ introduced a bootstrap approach to address the issue of heterogeneity under parameter-based MASEM. They suggested that their method could be used to quantify the uncertainty of the population parameters and the model fit of the structural equation models. Cheung ${ }^{68}$ corrected some errors in their paper and extended their simulation studies and found that Yu et al.'s approach could only be used to address the uncertainty of the population parameters, while the results on the model fit are misleading. Future work is required to find the best ways to address the issue of heterogeneity in MASEM.

Recently, MASEM has been introduced in mainstream meta-analysis and applied fields. For example, a special issue of Research Synthesis Methods ${ }^{69}$ discusses several recent methodological advances in MASEM. Hedges ${ }^{58}$ and Yuan, ${ }^{70}$ both well known in their areas of expertise (meta-analysis and SEM), were invited to comment on the contributed articles. Another indicator is that MASEM is occasionally featured in applied fields. ${ }^{71},{ }^{72},{ }^{73},{ }^{74}$ Many researchers are now aware of the power of MASEM to test theoretical models from a pool of empirical studies.

\section{Development of SEM-Based Meta-Analysis}

Another direction in the combining of meta-analysis and SEM is through SEM-based 
meta-analysis. ${ }^{44}$ It is well-known in the SEM community that many multivariate techniques such as the general linear model, item response theory, and multilevel model, can be considered special cases of SEM. Since my graduate studies, I have been fascinated with the question, “Can meta-analytic models be structural equation models?” This problem motivated me to work on SEM-based meta-analysis.

Inspired by Mehta and Neale, ${ }^{75}$ I realized that a study in a meta-analysis could be treated as a subject in a structural equation model. ${ }^{76}$ It then appeared clear to me that many concepts in meta-analysis and SEM share the same statistical model. For example, the observed effect sizes in a meta-analysis can be treated as the observed scores in a structural equation model; the population effect sizes in a meta-analysis can be interpreted as the latent scores in a structural equation model; and known sampling variances in a meta-analysis can be treated as fixed measurement errors in a structural equation model. The similarities between a meta-analytic model and a structural equation model make it possible to use SEM packages, e.g., Mplus ${ }^{44}$ and Stata, ${ }^{77}$ to conduct a meta-analysis. One direct benefit of formulating meta-analytic models as structural equation models is that techniques in SEM, such as handling missing data, non-independent data, and robust statistics, are directly available to researchers conducting a meta-analysis.

SEM-based meta-analysis was later extended to multivariate meta-analysis ${ }^{78}$ and three-level meta-analysis. ${ }^{79}$ Recently, this approach has been extended to address the heterogeneity of effect sizes ${ }^{80}$ and to network meta-analysis. ${ }^{81}$ SEM-based meta-analysis allows researchers to address many complicated research questions that are beyond the scope of a conventional meta-analysis. For example, Shadish and others ${ }^{26},{ }^{82}$ were interested in testing causal models on study characteristics and several population effect sizes. That is, there are "causal” models among population effect sizes. As population effect sizes are unobserved, researchers usually use observed effect sizes to approximate population effect sizes. This is not ideal, as population effect sizes are different from observed effect sizes. SEM-based meta-analysis treats population effect sizes as latent variables that can be used as both independent and dependent variables in model fitting (Section 5.6 of Cheung). ${ }^{44}$ Relationships among the population effect sizes can be hypothesized and empirically tested.

\section{Conclusion and Future Directions}

This paper summarizes my personal views on the history of synthesizing metaanalysis and SEM. Given the short history of MASEM, I believe that its best days are yet to come. Although meta-analysis has been very successfully used to synthesize research, I argue that it can also be valuable for analyzing primary data. For instance, meta-analysis can be valuable as a general framework for combining information in the era of Big Data. ${ }^{83}$ One 
standard approach to handling big data is the MapReduce framework and its variations. ${ }^{84},{ }^{85},{ }^{86}$ The central idea in the MapReduce framework is to split a large dataset into smaller pieces, which are then analyzed independently. The individual results are combined to form the final result. This framework is usually limited to the handling of such simple statistics as mean and counts. It remains unclear how it can be extended to general data analyses and model fitting.

Cheung and $\mathrm{Jak}^{87}$ proposed a split/analyze/meta-analyze (SAM) approach to analyzing big data by modifying the above framework using meta-analytic models to pool results from the individual pieces. They showed how the MASEM and the SEM-based metaanalyses could be integrated into this framework to conduct regression, mediation analysis, confirmatory factor analysis, and even structural equation modeling with big data.

Thus far, this paper has only focused on "standard” MASEM, that is, meta-analyzing correlation matrices for the purpose of fitting structural equation models, and on SEM-based meta-analysis. As reviewers have suggested, there are several allied topics that do not fall exactly into these two categories. It will be valuable to mention some of these developments so that readers are aware of how they are linked to the MASEM picture. Some researchers, e.g., Hafdahl ${ }^{88}, 54$, are mainly interested in improving the methodology for combining correlation matrices without fitting any structural equation models. It will be interesting to see how these procedures can be extended to fit structural equation models. Another topic is the combination of exploratory factor analysis and meta-analysis, which is useful when researchers have no idea of how the observed variables and latent constructs are related. Hafdah $^{49}$ provided early treatment of this topic, while Gnambs and Staufenbiel ${ }^{89}$ presented some recent developments on this topic. The last topic is the synthesis of regression coefficients. Correlation matrices are usually necessary inputs for MASEM. However, it may occasionally not be feasible to extract correlation matrices from published studies. Some researchers, e.g., Aloe and his colleagues, ${ }^{60},{ }^{61},{ }^{62},{ }^{90}$, have proposed using the partial effect size when summarizing research findings in regression models. Although the information provided by the partial effect size is not as rich as that in MASEM, it provides a viable alternative when MASEM cannot be used.

To conclude, I hope that this paper may stimulate more interest in applying metaanalytic thinking to other research domains. Meta-analysis has the potential to be a general tool for combining information in both primary research and research synthesis. We may also extend the current meta-analytic models by integrating ideas from other statistical techniques. 


\section{References}

1. Glass GV. Primary, secondary, and meta-analysis of research. Educ Res. 1976;5(10):3-8. doi:10.2307/1174772

2. Shadish WR. Introduction to the special issue on the origins of modern meta-analysis. Res Synth Methods. 2015;6(3):219-220. doi:10.1002/jrsm.1148

3. Glass GV. Meta-analysis at middle age: A personal history. Res Synth Methods. 2015;6(3):221-231. doi:10.1002/jrsm.1133

4. Rosenthal R. Reflections on the origins of meta-analysis. Res Synth Methods. 2015;6(3):240-245. doi:10.1002/jrsm.1135

5. Schmidt FL. History and development of the Schmidt-Hunter meta-analysis methods. Res Synth Methods. 2015;6(3):232-239. doi:10.1002/jrsm.1134

6. Cappelleri JC, Ingerick M. A conversation with Joseph Lau. Res Synth Methods. 2015;6(1):2-20. doi:10.1002/jrsm.1116

7. $\quad$ Shadish WR, Lecy JD. The meta-analytic big bang. Res Synth Methods. 2015;6(3):246264. doi:10.1002/jrsm.1132

8. Bentler PM. Structural modeling and psychometrika: An historical perspective on growth and achievements. Psychometrika. 1986;51(1):35-51. doi:10.1007/BF02293997

9. Jöreskog KG. A general approach to confirmatory maximum likelihood factor analysis. Psychometrika. 1969;34(2):183-202. doi:10.1007/BF02289343

10. Kline RB. Principles and Practice of Structural Equation Modeling. 4th ed. New York: Guilford Press; 2016.

11. Cudeck R, Du Toit S, Sörbom D, eds. Structural Equation Modeling: Present and Future: A Festschrift in Honor of Karl Jöreskog. Chicago: Scientific Software; 2001.

12. Matsueda RL. Key advances in the history of structural equation modeling. In: Hoyle RH, ed. Handbook of Structural Equation Modeling. New York: Guilford Press; 2012:342.

13. American Psychological Association. Peter M. Bentler Award for Distinguished Scientific Applications of Psychology. Am Psychol. 2007;62(8):769-782. doi:10.1037/0003-066X.62.8.769

14. American Psychological Association. Karl G. Jöreskog Award for Distinguished Scientific Applications of Psychology. Am Psychol. 2007;62(8):768-769. doi:10.1037/0003-066X.62.8.768

15. Becker BJ, Schram CM. Examining explanatory models through research synthesis. In: Cooper H, Hedges LV, eds. The Handbook of Research Synthesis. New York: Russell Sage Foundation; 1994:357-381.

16. Becker BJ. Model-based meta-analysis. In: Cooper H, Hedges LV, Valentine JC, eds. The Handbook of Research Synthesis and Meta-Analysis. 2nd ed. New York: Russell Sage Foundation; 2009:377-395. 
17. Colquitt JA, LePine JA, Noe RA. Toward an integrative theory of training motivation: A meta-analytic path analysis of 20 years of research. J Appl Psychol. 2000;85(5):678707. doi:10.1037/0021-9010.85.5.678

18. Becker G. The meta-analysis of factor analyses: An illustration based on the cumulation of correlation matrices. Psychol Methods. 1996;1(4):341-353. doi:10.1037/1082989X.1.4.341

19. Bushman BJ, Cooper HM, Lemke KM. Meta-analysis of factor analyses: An illustration using the Buss-Durkee Hostility Inventory. Pers Soc Psychol Bull. 1991;17(3):344-349. doi:10.1177/0146167291173015

20. Hom PW, Caranikas-Walker F, Prussia GE, Griffeth RW. A meta-analytical structural equations analysis of a model of employee turnover. J Appl Psychol. 1992;77(6):890909. doi:10.1037/0021-9010.77.6.890

21. Eby LT, Freeman DM, Rush MC, Lance CE. Motivational bases of affective organizational commitment: A partial test of an integrative theoretical model. $J$ Occup Organ Psychol. 1999;72(4):463-483. doi:10.1348/096317999166798

22. Conway JM. Distinguishing contextual performance from task performance for managerial jobs. J Appl Psychol. 1999;84(1):3-13. doi:10.1037/0021-9010.84.1.3

23. Tett RP, Meyer JP. Job satisfaction, organizational commitment, turnover intention, and turnover: Path analyses based on meta-analytic findings. Pers Psychol. 1993;46(2):259293. doi:10.1111/j.1744-6570.1993.tb00874.x

24. Sheng Z, Kong W, Cortina JM, Hou S. Analyzing matrices of meta-analytic correlations: Current practices and recommendations. Res Synth Methods. 2016;7(2):187-208. doi:10.1002/jrsm.1206

25. Becker BJ. Using results from replicated studies to estimate linear models. J Educ Stat. 1992;17(4):341-362. doi:10.3102/10769986017004341

26. Shadish WR. Meta-analysis and the exploration of causal mediating processes: A primer of examples, methods, and issues. Psychol Methods. 1996;1(1):47-65. doi:10.1037/1082-989X.1.1.47

27. Viswesvaran C, Ones DS. Theory testing: Combining psychometric meta-analysis and structural equations modeling. Pers Psychol. 1995;48(4):865-885. doi:10.1111/j.17446570.1995.tb01784.x

28. Harris MJ, Rosenthal R. Mediation of interpersonal expectancy effects: 31 metaanalyses. Psychol Bull. 1985;97(3):363-386. doi:10.1037/0033-2909.97.3.363

29. Schmidt FL, Hunter JE, Outerbridge AN. Impact of job experience and ability on job knowledge, work sample performance, and supervisory ratings of job performance. $J$ Appl Psychol. 1986;71(3):432-439. doi:10.1037//0021-9010.71.3.432

30. Premack SL, Hunter JE. Individual unionization decisions. Psychol Bull. 1988;103(2):223-234. doi:10.1037/0033-2909.103.2.223

31. Becker BJ. Multivariate meta-analysis: Contributions of Ingram Olkin. Stat Sci. 2007;22(3):401-406. doi:10.1214/07-STS239 
32. Jackson D, Riley R, White IR. Multivariate meta-analysis: Potential and promise. Stat Med. 2011;30(20):2481-2498. doi:10.1002/sim.4172

33. Marsh HW. Pairwise deletion for missing data in structural equation models:

Nonpositive definite matrices, parameter estimates, goodness of fit, and adjusted sample sizes. Struct Equ Model Multidiscip J. 1998;5(1):22-36. doi:10.1080/10705519809540087

34. Savalei V, Bentler PM. A statistically justified pairwise ML method for incomplete nonnormal data: A comparison with direct ML and pairwise ADF. Struct Equ Model Multidiscip J. 2005;12(2):183-214. doi:10.1207/s15328007sem1202_1

35. Cudeck R. Analysis of correlation matrices using covariance structure models. Psychol Bull. 1989;105(2):317-327. doi:10.1037/0033-2909.105.2.317

36. Enders CK. Applied Missing Data Analysis. New York: Guilford Press; 2010.

37. Furlow CF, Beretvas NS. Meta-analytic methods of pooling correlation matrices for structural equation modeling under different patterns of missing data. Psychol Methods. 2005;10(2):227-254. doi:10.1037/1082-989X.10.2.227

38. Cheung MW-L, Chan W. Meta-analytic structural equation modeling: A two-stage approach. Psychol Methods. 2005;10(1):40-64. doi:10.1037/1082-989X.10.1.40

39. Jak S, Cheung MW-L. Accounting for missing correlation coefficients in fixed-effects MASEM. Multivar Behav Res. 2018;53(1):1-14. doi:10.1080/00273171.2017.1375886

40. Becker BJ. Corrections to "Using results from replicated studies to estimate linear models.” J Educ Behav Stat. 1995;20(1):100-102. doi:10.2307/1165390

41. Hunter JE, Schmidt FL. Methods of Meta-Analysis: Correcting Error and Bias in Research Findings. 2nd ed. Thousand Oaks, CA: Sage; 2004.

42. Rosopa PJ, Kim B. Robustness of statistical inferences using linear models with metaanalytic correlation matrices. Hum Resour Manag Rev. 2017;27(1):216-236. doi:10.1016/j.hrmr.2016.09.012

43. Hedges LV, Olkin I. Statistical Methods for Meta-Analysis. Orlando, FL: Academic Press; 1985.

44. Cheung MW-L. Meta-Analysis: A Structural Equation Modeling Approach. Chichester, West Sussex: John Wiley \& Sons, Inc.; 2015.

45. Cheung MW-L. Meta-analysis for structural equation modeling: A two-stage approach. 2002. http://search.proquest.com/docview/276369475/abstract/13CA3F7BD5A3508CDC7/16 ?accountid=13876. Accessed March 6, 2013.

46. Browne MW. Asymptotically distribution-free methods for the analysis of covariance structures. Br J Math Stat Psychol. 1984;37(1):62-83. doi:10.1111/j.20448317.1984.tb00789.x

47. Cheung MW-L. Fixed-effects meta-analyses as multiple-group structural equation models. Struct Equ Model Multidiscip J. 2010;17(3):481-509. 
doi:10.1080/10705511.2010.489367

48. Cheung MW-L, Chan W. A two-stage approach to synthesizing covariance matrices in meta-analytic structural equation modeling. Struct Equ Model Multidiscip J. 2009;16(1):28-53. doi:10.1080/10705510802561295

49. Hafdahl AR. Multivariate meta-analysis for exploratory factor analytic research. 2001. http://search.proquest.com/docview/251408097/abstract?accountid=13876. Accessed March 6, 2013.

50. Cheung SF. Examining solutions to two practical issues in meta-analysis: Dependent correlations and missing data in correlation matrices. 2000.

51. Cheung MW-L. Fixed- and random-effects meta-analytic structural equation modeling: Examples and analyses in R. Behav Res Methods. 2014;46(1):29-40. doi:10.3758/s13428-013-0361-y

52. Olkin I, Siotani M. Asymptotic distribution of functions of a correlation matrix. In: Ideka S, ed. Essays in Probability and Statistics. Tokyo: Shinko Tsusho; 1976:235-251.

53. Becker BJ, Fahrbach K. A comparison of approaches to the synthesis of correlation matrices. Presented at the: The annual meeting of the American Educational Research Association; April 1994; New Orleans, L.A.

54. Hafdahl AR. Combining heterogeneous correlation matrices: Simulation analysis of fixed-effects methods. J Educ Behav Stat. 2008;33(4):507-533. doi:10.3102/1076998607309472

55. Cheung MW-L. metaSEM: an R package for meta-analysis using structural equation modeling. Front Psychol. 2015;5(1521). doi:10.3389/fpsyg.2014.01521

56. R Development Core Team. R: A Language and Environment for Statistical Computing. Vienna, Austria; 2018. http://www.R-project.org/.

57. Jak S. Meta-Analytic Structural Equation Modelling. Cham: Springer International Publishing; 2015.

58. Hedges LV. Applying meta-analysis to structural equation modeling. Res Synth Methods. 2016;7(2):209-214. doi:10.1002/jrsm.1214

59. Cheung MW-L, Cheung SF. Random-effects models for meta-analytic structural equation modeling: Review, issues, and illustrations. Res Synth Methods. 2016;7(2):140155. doi:10.1002/jrsm.1166

60. Aloe AM, Becker BJ. An effect size for regression predictors in meta-analysis. J Educ Behav Stat. 2012;37(2):278-297. doi:10.3102/1076998610396901

61. Aloe AM, Thompson CG. The synthesis of partial effect sizes. J Soc Soc Work Res. 2013;4(4):390-405. doi:10.5243/jsswr.2010.

62. Aloe AM. An empirical investigation of partial effect sizes in meta-analysis of correlational data. J Gen Psychol. 2014;141(1):47-64. doi:10.1080/00221309.2013.853021 
63. Hafdahl AR. Meta-analysis for functions of heterogeneous multivariate effect sizes. 2009. http://openscholarship.wustl.edu/etd/439.

64. Becker BJ, Wu M-J. The synthesis of regression slopes in meta-analysis. Stat Sci. 2007;22(3):414-429. doi:10.1214/07-STS243

65. Paul PA, Lipps PE, Madden LV. Meta-analysis of regression coefficients for the relationship between fusarium head blight and deoxynivalenol content of wheat. Phytopathology. 2006;96(9):951-961. doi:10.1094/PHYTO-96-0951

66. Gasparrini A, Armstrong B, Kenward MG. Multivariate meta-analysis for non-linear and other multi-parameter associations. Stat Med. 2012;31(29):3821-3839. doi:10.1002/ $\operatorname{sim} .5471$

67. Yu J (Joya), Downes PE, Carter KM, O’Boyle EH. The problem of effect size heterogeneity in meta-analytic structural equation modeling. J Appl Psychol. 2016;101(10):1457-1473. doi:10.1037/apl0000141

68. Cheung MW-L. Issues in solving the problem of effect size heterogeneity in metaanalytic structural equation modeling: a commentary and simulation study on $\mathrm{Yu}$, Downes, Carter, and O’Boyle (2016). J Appl Psychol. 2017. https://psyarxiv.com/37p2z. Accessed December 12, 2017.

69. Cheung MW-L, Hafdahl AR. Special issue on meta-analytic structural equation modeling: Introduction from the guest editors. Res Synth Methods. 2016;7(2):112-120. doi:10.1002/jrsm.1212

70. Yuan K-H. Meta analytical structural equation modeling: Comments on issues with current methods and viable alternatives. Res Synth Methods. 2016;7(2):215-231. doi:10.1002/jrsm.1213

71. Bergh DD, Aguinis H, Heavey C, et al. Using meta-analytic structural equation modeling to advance strategic management research: Guidelines and an empirical illustration via the strategic leadership-performance relationship. Strateg Manag J. 2016;37(3):477-497. doi:10.1002/smj.2338

72. Cheung MW-L, Hong RY. Applications of meta-analytic structural equation modelling in health psychology: examples, issues, and recommendations. Health Psychol Rev. 2017;11(3):265-279. doi:10.1080/17437199.2017.1343678

73. Landis RS. Successfully combining meta-analysis and structural equation Modeling: Recommendations and strategies. J Bus Psychol. 2013;28(3):251-261. doi:10.1007/s10869-013-9285-X

74. Tang RW, Cheung MW-L. Testing IB theories with meta-analytic structural equation modeling: The TSSEM approach and the Univariate-r approach. Rev Int Bus Strategy. 2016;26(4):472-492. doi:10.1108/RIBS-04-2016-0022

75. Mehta PD, Neale MC. People are variables too: Multilevel structural equations modeling. Psychol Methods. 2005;10(3):259-284. doi:10.1037/1082-989X.10.3.259

76. Cheung MW-L. A model for integrating fixed-, random-, and mixed-effects metaanalyses into structural equation modeling. Psychol Methods. 2008;13(3):182-202. doi:10.1037/a0013163 
77. Palmer TM, Sterne JAC. Fitting fixed and random effects meta-analysis models using structural equation modeling with the sem and gsem commands. Stata J. 2014;15(3):645-671.

78. Cheung MW-L. Multivariate meta-analysis as structural equation models. Struct Equ Model Multidiscip J. 2013;20(3):429-454. doi:10.1080/10705511.2013.797827

79. Cheung MW-L. Modeling dependent effect sizes with three-level meta-analyses: A structural equation modeling approach. Psychol Methods. 2014;19(2):211-229. doi:10.1037/a0032968

80. Schoemann AM. Using multiple group modeling to test moderators in meta-analysis. Res Synth Methods. 2016;7(4):387-401. doi:10.1002/jrsm.1200

81. Tu Y-K, Wu Y-C. Using structural equation modeling for network meta-analysis. BMC Med Res Methodol. 2017;17(104). doi:10.1186/s12874-017-0390-9

82. Shadish WR, Sweeney RB. Mediators and moderators in meta-analysis: There's a reason we don't let dodo birds tell us which psychotherapies should have prizes. $J$ Consult Clin Psychol. 1991;59(6):883-893. doi:10.1037/0022-006X.59.6.883

83. Johnson BT. Meta-analysis: The original "Big Data." http://meta-analysis.ning.com/profiles/blogs/meta-analysis-the-original-big-data. Published August 19, 2013. Accessed December 19, 2017.

84. Dean J, Ghemawat S. MapReduce: Simplified data processing on large clusters. Commun ACM. 2008;51(1):107-113. doi:10.1145/1327452.1327492

85. Matloff N. Software Alchemy: Turning complex statistical computations into embarrassingly-parallel ones. J Stat Softw. 2016;71(4):1-15. doi:10.18637/jss.v071.i04

86. Wickham H. The split-apply-combine strategy for data analysis. J Stat Softw. 2011;40(1):1-29.

87. Cheung MW-L, Jak S. Analyzing big data in psychology: A split/analyze/meta-analyze approach. Front Psychol. 2016;7(738). doi:10.3389/fpsyg.2016.00738

88. Hafdahl AR. Combining correlation matrices: Simulation analysis of improved fixedeffects methods. J Educ Behav Stat. 2007;32(2):180-205. doi:10.3102/1076998606298041

89. Gnambs T, Staufenbiel T. Parameter accuracy in meta-analyses of factor structures. Res Synth Methods. 2016;7(2):168-186. doi:10.1002/jrsm.1190

90. Aloe AM. Inaccuracy of regression results in replacing bivariate correlations. Res Synth Methods. 2015;6(1):21-27. doi:10.1002/jrsm.1126 